\title{
Experimental demonstration of compact spoof localized surface plasmons
}

\author{
Di BaO ${ }^{1,2}$, Khalid Z. Rajab ${ }^{3}$, Wei XIANg Jiang ${ }^{1,2}$, QIANg Cheng ${ }^{1,2}$, Zhen \\ LIAO ${ }^{1,2}$ AND TIE JUN CUI ${ }^{1,4 *}$ \\ ${ }^{1}$ State Key Laboratory of Millimeter Waves, School of Information Science and Engineering, Southeast University, Nanjing 210096, China \\ ${ }^{2}$ Synergetic Innovation Center of Wireless Communication Technology, Southeast University, Nanjing 210096, China \\ ${ }^{3}$ School of Electronic Engineering and Computer Science, Queen Mary University of London, London E1 4NS, UK \\ ${ }^{4}$ Cooperative Innovation Centre of Terahertz Science, No. 4, Section 2, North Jianshe Road, Chengdu 610054, China \\ *Email: tjcui@seu.edu.cn
}

Received XX Month XXXX; revised XX Month, XXXX; accepted XX Month XXXX; posted XX Month XXXX (Doc. ID XXXXX); published XX Month XXXX

In recent years, the study of generating and detecting localized surface plasmons (LSPs) has been expanded from the optical regime to microwave regime. In this work, the compact spoof LSPs are introduced through both numerical simulations and near-field measurements. It is observed that the compact LSP structure could effectively reduce the resonant frequency with stronger resonance strength $\left(\mathrm{G}_{d b s m}\right)$ and higher $\mathbf{Q}$ factor. Both electric near-field and surface-current distributions are monitored to examine the resonance processes of the LSP particle. (C) 2016 Optical Society of America

OCIS codes: (250.5403) Plasmonics; (160.3918) Metamaterials;

(240.6680) Surface plasmons

http://dx.doi.org/XXXXXXXXXXXXXXXX

Surface plasmons are electron oscillations along the interface between dielectrics and metals at optical frequencies. With the possibility of deep subwavelength confinement and field enhancement, surface plasmons are of intensive interest [1-3] with potential applications in bio-sensing and spectroscopic detection. However, at microwave and terahertz regions, metals behave similarly to perfect electric conductors, and therefore surface plasmons can no longer be excited naturally. Fortunately, it has been recognized that periodically structured metals support surface waves which mimic the surface plasmons at these lower frequencies [4]. Following this theory, designer or spoof surface plasmon polaritons (SPPs) and electric and magnetic localized surface plasmons (LSPs) have been introduced and experimentally measured [5-10]. Recently, several modified LSP structures have been proposed for further insight into this field. For example, LSPs in closed textured cavities were designed and studied numerically in two dimensions [11]. Recently, a metal-insulator-metal (MIM) ring resonator [12] and the complementary corrugated ring structure [13] were designed and fed with microstrip line. The properties of the complementary metallic spiral structure was examined by the Babinet's principle, where the measured electric field distributions of the complementary metallic spiral structure could be applied to map the magnetic field distributions of a corresponding mode in the original metallic spiral structure $[14,15]$.

In this letter, we design and experimentally demonstrate a compact spoof LSP structure which has smaller electric dimensions, stronger resonance and higher quality $(\mathrm{Q})$ factor. The conformation of the proposed structure is the complementary geometry of the ultrathin spoof LSP [9]. Instead of analysing with the Babinet's principle, we still study the electric resonance, and the structure revealed much lower resonant frequency than both the original structure [9] and the corrugated ring structure [13]. Meanwhile, the electric LSP resonance $\mathrm{Q}$ factor is much higher than that in previous designs. Both electric field distributions and surface current distributions are monitored to analyse the resonant properties.
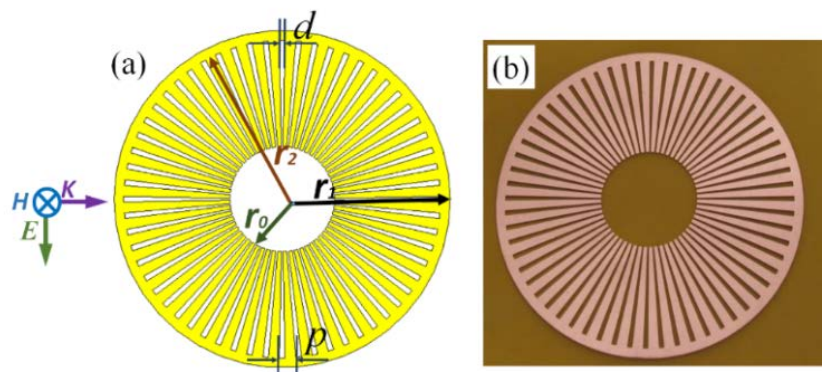

Fig. 1. (a) The schematic configuration of the compact LSP structure. (b) The photo of the compact LSP.

The schematic configuration of the proposed compact spoof LSP structure and its photo are shown in Figures 1(a) and 1(b), respectively. The original structure, to which it is complementary, is shown in Fig. 2(a). The thin copper structure with thickness $0.018 \mathrm{~mm}$ is etched on an ultrathin layer of polyimide with the thickness of 0.1 $\mathrm{mm}$ and dielectric constant of 3.5. The variables in Fig. 1(a) are chosen as $r_{0}=3.39 \mathrm{~mm}, r_{1}=12 \mathrm{~mm}, r_{2}=11.3 \mathrm{~mm}, d=0.4 \mathrm{~mm}$, and $p=1.1833 \mathrm{~mm}$. In this new design, the electric current path is longer due to the greater 
circumference as compared to the original structure; thus a lower working frequency band is expected. The sample of the original LSP structure in Fig. 2(a) is complementary to the metal structure in Fig. 1 (a), and for better comparison, a corrugated ring structure [13] is also fabricated as shown in Fig. 2(b), where a metallic ring with inner radius of $7.345 \mathrm{~mm}$ and width of $0.4 \mathrm{~mm}$ is placed in the middle of the structure to connect all the rods. The rods have the same dimensions as those in Fig. 2(a).

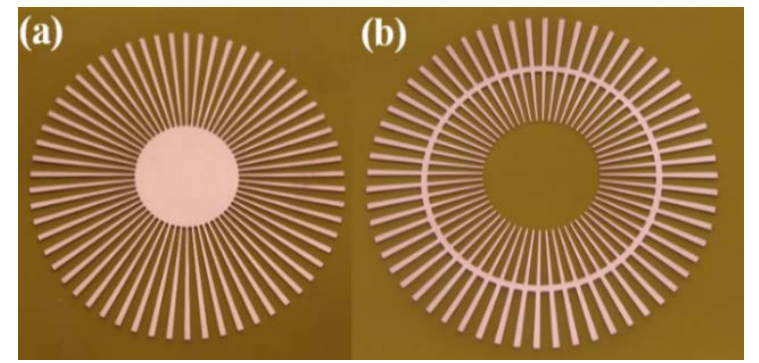

Fig. 2. The photo of the fabrication sample of (a) the original spoof LSP structure, and (b) the corrugated ring structure.

To verify the performance of the designed compact LSP structure, full wave numerical simulations were first performed using the commercial software package, CST Microwave Studio. In the simulation, a TEM polarized plane-wave is launched from the left with magnetic wave perpendicular to the surface of the sample as shown in Fig. 1(a). Both radar cross section (RCS) and absorption cross section (ACS) spectra could be obtained by numerical simulations in CST. In order to quantitatively evaluate the coupling strength of the electromagnetic waves with the sample under test, the parameter $\mathrm{G}_{d B s m}$ is employed, which is defined as:

$G_{d B s m}=10 \log _{10}\left(\frac{E C S}{m^{2}}\right)=10 \log _{10}\left(\frac{R C S+A C S}{m^{2}}\right)$.

where $E C S=R C S+A C S$ is the extinction cross section. The calculated spectra of $\mathrm{G}_{d B s m}$ for the proposed compact LSP structure is plotted in Fig. 3 along with those of the original LSP and the corrugated ring LSP structures. Q factor, which could be used to evaluate the underdamping property of the resonator, can now be calculated based on the $\mathrm{G}_{d B s m}$ spectra from the following equation:

$$
Q=\frac{f_{0}}{f_{2}-f_{1}}
$$

Where $f_{0}$ is the center frequency, $f_{1}$ and $f_{2}$ are the half-power frequencies.

For better comparison, the detailed data in the $\mathrm{G}_{d B s m}$ spectra and $\mathrm{Q}$ factors are listed in Table 1 . It could be observed that the resonance frequency of the basic mode, dipole mode, in the compact unit is significantly reduced from $4.43 \mathrm{GHz}$ to $3.2 \mathrm{GHz}$, with the corresponding wavelength increased from $67.67 \mathrm{~mm}$ to $93.69 \mathrm{~mm}$ by $38.45 \%$. Meanwhile, the dipole mode resonant strength, $\mathrm{G}_{d B s m}$, of the compact particle is $-56.76 \mathrm{dBsm}$, which is higher than the original structure by $5.13 \mathrm{dBsm}$. The $\mathrm{Q}$ factor of the compact LSP structure is 17.81, while the $Q$ value of the original one is only 4.9. Thus, the complementary structure proposed here has smaller electrical size and higher basic mode resonance strength than the original design.

The frequencies of quadrupole and hexapole mode are also reduced from 6.03 and $6.96 \mathrm{GHz}$ in the original structure to 5.28 and $6.34 \mathrm{GHz}$ in the compact structure, while increased to 6.71 and 8.88 $\mathrm{GHz}$ in the corrugated ring structure. The resonance strengthes of the quadruple mode are both around - $60 \mathrm{dBsm}$, but the $\mathrm{Q}$ factor increased from 23.3 into 146.5. The resonance strength of the quadruple mode is decreased $18.22 \mathrm{dBsm}$ into $-78.61 \mathrm{dBsm}$. However, the hexapole mode
Q factor of the compact structure is 634, while the original one is 198.84, which is also the highest $Q$ factor in all the three modes of the three structures.

The simulated and measured near field distributions are plotted in Figs. 4(a-f), where all the dipole, quadruple and hexapole modes could be clearly observed in both simulations and experiments, and agreed quite well with the calculated peaks in $\mathrm{G}_{d B s m}$ spectra.

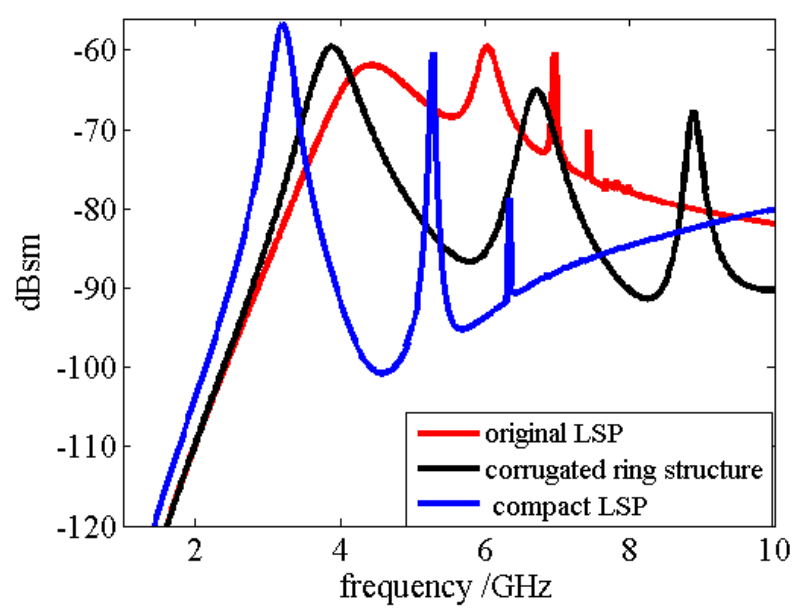

Fig. 3. The calculated GdBsm spectra based on ECS of the localized LSP structures.

Table 1 Comparison of the three structures

\begin{tabular}{llll}
\hline & $\begin{array}{l}\text { Original } \\
\text { structure }\end{array}$ & $\begin{array}{l}\text { Corrugated ring } \\
\text { structure }\end{array}$ & $\begin{array}{l}\text { Compact } \\
\text { structure }\end{array}$ \\
\hline $\mathrm{f}$ of $\mathrm{M}_{1} / \mathrm{GHz}$ & 4.43 & 3.89 & 3.2 \\
$\mathrm{G}_{d B s m}$ of $\mathrm{M}_{1} / d B s m$ & -61.89 & -59.62 & -56.76 \\
$\mathrm{Q}$ of $\mathrm{M}_{1}$ & 4.90 & 9.13 & 17.81 \\
\hline $\mathrm{f}$ of $\mathrm{M}_{2} / \mathrm{GHz}$ & 6.03 & 6.71 & 5.28 \\
$\mathrm{G}_{d B s m}$ of $\mathrm{M}_{2} / d B s m$ & -59.54 & -65.05 & -60.23 \\
$\mathrm{Q}$ of $\mathrm{M}_{2}$ & 23.30 & 22.17 & 146.5 \\
\hline $\mathrm{f}$ of $\mathrm{M}_{3} / \mathrm{GHz}$ & 6.96 & 8.88 & 6.34 \\
$\mathrm{G}_{d B s m}$ of $\mathrm{M}_{3} / d B s m$ & -60.39 & -67.74 & -78.61 \\
$\mathrm{Q}$ of $\mathrm{M}_{3}$ & 198.84 & 67.81 & 634
\end{tabular}

$* \mathrm{M}_{1}$ is the first-order mode (dipole), $\mathrm{M}_{2}$ is the second-order mode (quadruple), and $\mathrm{M}_{3}$ is the third-order mode (hexapole). $\mathrm{f}$ is the resonant frequency.

The resonance LSP modes are standing waves in the ring, and these phenomena could be compared with microwave ring resonators [16] which have been adopted to analyze the resonance processes in split-ring resonators (SRRs) [17]. As shown in Fig. 4(g), the surface currents of the dipole modes in the compact LSP structure are excited by the penetrated magnetic waves in full-wavelength resonance, and the currents mainly resonances as a pair of in-phase surface current along the periphery annular path in the two sides of the metallic structure. In this case, the circumference of the surface current path equals to the integral multiple of the guided wavelength, $\lambda_{g}$. This relation could be expressed as:

$$
2 \pi r_{g}=n \lambda_{g}, \quad \text { for } n=1,2,3 \ldots
$$


where $r_{g}$ is the guide radius of the surface current, and $n$ is the mode number. In the dipole mode of Figs. 4(a) and (g), the structure has positive charges on the left side and negative charges on the right. Thus the electric fields are oscillated in opposite phase in the left and right halves, as demonstrated in simulation in Fig. 4(a) and experiment is Fig. 4(d).
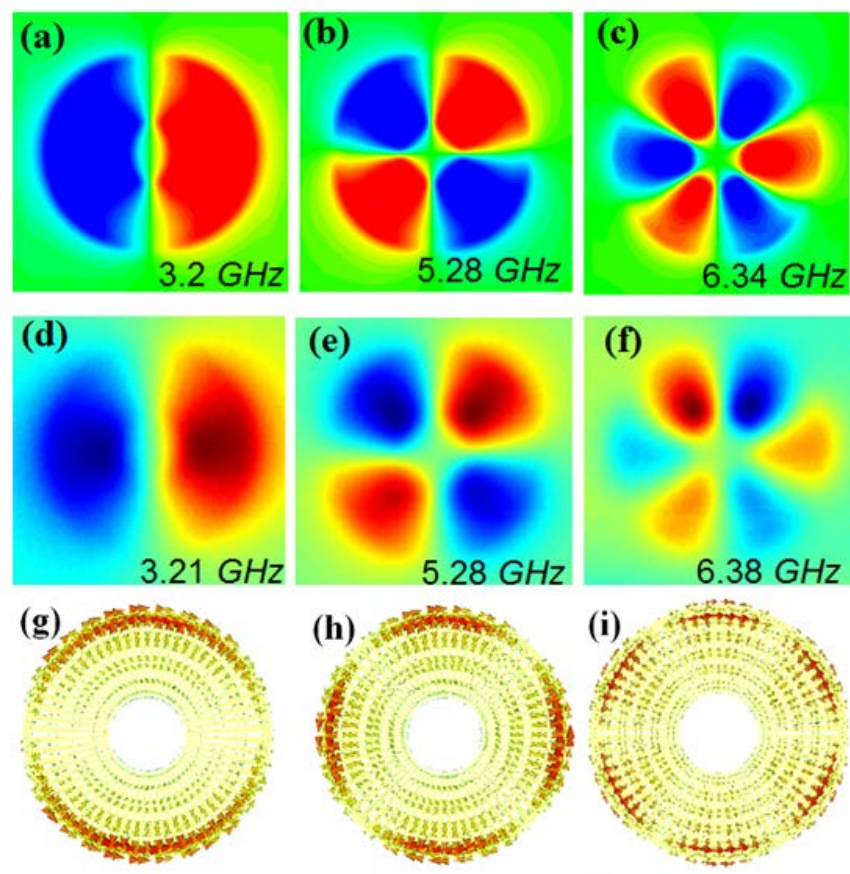

Fig. 4. The simulated (a-c) and measured (d-f) near field distributions, and surface current distributions (g-i) of the compact LSP structure of $(\mathrm{a}, \mathrm{d}, \mathrm{g})$ dipole, (b,e,h) quadruple, and (c,f,i) hexapole mode.

The surface current of the quadruple mode and hexapole mode of the compact LSP structure are also monitored and plotted in Figs. 4(h) and (i), respectively. It could be observed that there are two pairs of surface currents in the quadruple mode and three pairs of surface currents in the hexapole mode. Accordingly, there should be two pairs and three pairs of electric fields oscillated in opposite phase, as shown in simulation results in Figs. 4(b) and (c), and measured results in Figs. 4(e) and (f). It is worth noting that in this compact LSP structure, the width of the outermost ring is $0.7 \mathrm{~mm}$. Due to its narrow profile the path of the surface current is determined by the ring. When $r_{2}$ is much larger than $r_{1}$, the ring with noticeable width will lead the surface current to be distributed throughout the ring, with the main current travelling along the inner edges. In this case, the equivalent surface current path radius will be between $r_{1}$ and $r_{2}$, and hence the $\mathrm{M}_{1}$ frequency is reduced slightly to $2.99 \mathrm{GHz}$. However, such a design has much larger electric dimension and weaker resonances compared to the compact LSP structure. So in the design of this compact LSP particle, the inner radius of the ring, $r_{2}$, is the most important parameter for determining the location of the surface current.
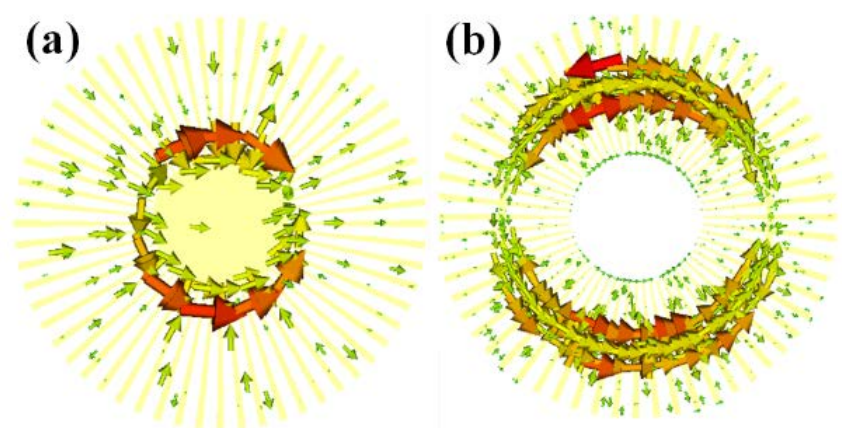

Fig. 5. The surface current distribution of the dipole modes in the LSP structures. (a) The original structure. (b) The corrugated ring structure.

The surface currents of the original LSP structure and corrugated ring structure are monitored and plotted in Fig. 5 to study the resonance property. As shown in Fig. 4(g), for the compact LSP structure, the resonance path is located at the periphery of the metallic structure, with the radius of $12 \mathrm{~mm}$, while in the original structure, the resonance path lies at the middle of the pattern with the radius of 3.7 $\mathrm{mm}$, as shown in Fig. 5(a). The resonance path of the corrugated ring structure lies between the compact LSP and original LSP structure, as shown in Fig. 5(b). With the increase of the resonance path, the frequency is decreased accordingly. However, due to the scattered surface current on the metallic rods, the resonant frequency is not directly inversely proportional to the radius of the surface current path.

Here in this letter, a compact metamaterial particle is designed which could support spoof localized SPP waves with smaller electrical dimension. Both numerical simulations and experiments are carried out to verify the performance of the structure. The resonance processes are investigated based on the near field and surface current distributions, and compared with the original LSP structure and the corrugated ring structure. The designed compact LSP structure possesses stronger resonant strength and higher $\mathrm{Q}$ factor, which has potential application in bio-sensing and ultrathin material measurement. The compact LSP structure is akin to dielectric inclusion in metals with void plasmons, while the original structure is similar to metallic nanoparticles with localized surface plasmons. So a combined structure with the compact LSP structure and the original LSP might lead to plasmon hybridization, as with that in metallic nanoshells [18]. More comprehensive research on hybridization structures is under study.

Funding. This work was supported in part from the National Natural Science Foundation of China under Grant Nos. 61302018, 61401089, 61571117, 61501112, 61501117 and 61138001, in part from the 111 Project under Grant No. 111-2-05, in part from the Foundation for the Author of National Excellent Doctoral Dissertation of the People's Republic of China under Grant No. 201444, and in part from the National Instrumentation Program (2013YQ200647)

\section{References}

1. W. L. Barnes, A. Dereux, and T. W. Ebbesen, Nature (London) 424, 824 (2003).

2. E. Ozbay, Science 311, 189 (2006).

3. M. Dragoman and D. Dragoman, Prog. Quantum Electron. 32, 1 (2008).

4. J. B. Pendry, L. Martin-Moreno, and F. J. Garcia-Vidal, Science 305, 847 (2004). 
5. F. J. Garcia-Vidal, L. Mart' In-Moreno, and J. B. Pendry, J. Opt. A: Pure Appl. Opt. 7, 597 (2005).

6. Q. Gan, Z. Fu, Y. J. Ding, and F. J. Bartoli, Phys. Rev. Lett. 100(25), 256803 (2008).

7. A. P. Hibbins, B. R. Evans, and J. R. Sambles, Science 308, 670 (2005).

8. X. P. Shen, T. J. Cui, D. Martin-Cano, and F. J. Garcia-Vidal, Proc. Natl. Acad. Sci. U.S.A. 110, 40 (2013)

9. X. P. Shen and T. J. Cui, Laser Photonics Rev. 8, 137 (2014)

10. P. A. Huidobro, X. P. Shen, J. Cuerda, E. Moreno, L. Martin-Moreno, F. J. Garcia-Vidal, T. J. Cui, and J. B. Pendry, Phys. Rev. X. 4, 021003 (2014)

11. Z. Li, B. Z. Xu, C. Q. Gu, P. P. Ning, L. L. Liu, Z. Y. Niu, and Y. J. Zhao, Appl. Phys. Lett. 104, 251601 (2014)

12. Y. J. Zhou, Q. Z. Xiao, B. J. Yang, Sci. Rep., 5,14819(2015)

13. B. J. Yang, Y. J. Zhou, Q. X. Xiao, Opt. Express, 23, 021434 (2015)

14. Z. Gao,F. Gao, Y. Zhang and B. Zhang, Appl. Phys. Lett., 107, 191103 (2015),

15. J. A. Kong, Electromagnetic Wave Theory, E M W Pub, (2000)

16. K. Chang, Microwave Ring Circuits and Antennas. New York: Wiley (1996)

17. E. A. Semouchkina, G. B. Semouchkin, M. Lanagan, and C. A. Randall, IEEE Trans. Microw.Theory Tech $\mathbf{5 3}$ 4(2005)

18. S. A. Maier, Plasmonics: fundamentals and applications, Springer, (2007) 


\section{References}

1. W. L. Barnes, A. Dereux, and T. W. Ebbesen, "Surface plasmon subwavelength optics", Nature (London) 424, 824 (2003).

2. E. Ozbay, "Plasmonics: Merging Photonics and Electronics at Nanoscale Dimensions", Science 311, 189 (2006).

3. M. Dragoman and D. Dragoman, "Plasmonics: Applications to nanoscale terahertz and optical devices", Prog. Quantum Electron. 32, 1 (2008).

4. J. B. Pendry, L. Martin-Moreno, and F. J. Garcia-Vidal, "Mimicking surface plasmons with structured surfaces", Science 305, 847 (2004).

5. F. J. Garcia-Vidal, L. Mart'ın-Moreno, and J. B. Pendry, "Surfaces with holes in them: new plasmonic metamaterials", J. Opt. A: Pure Appl. Opt. 7, S97 (2005).

6. Q. Gan, Z. Fu, Y. J. Ding, and F. J. Bartoli, “Ultrawide-bandwidth slow-light system based on THz plasmonic graded metallic grating structures," Phys. Rev. Lett. 100(25), 256803 (2008).

7. A. P. Hibbins, B. R. Evans, and J. R. Sambles, "Experimental verification of designer surface plasmons", Science 308, 670 (2005).

8. X. P. Shen, T. J. Cui, D. Martin-Cano, and F. J. Garcia-Vidal, "Conformal surface plasmons propagating on ultrathin and flexible films", Proc. Natl. Acad. Sci. U.S.A. 110, 40 (2013)

9. X. P. Shen and T. J. Cui, "Ultrathin plasmonic metamaterial for spoof localized surface plasmons", Laser Photonics Rev. 8, 137 (2014)

10. P. A. Huidobro, X. P. Shen, J. Cuerda, E. Moreno, L. Martin-Moreno, F. J. Garcia-Vidal, T. J. Cui, and J. B. Pendry, "Magnetic localized surface plasmons", Phys. Rev. X. 4, 021003 (2014)

11. Z. Li, B. Z. Xu, C. Q. Gu, P. P. Ning, L. L. Liu, Z. Y. Niu, and Y. J. Zhao, "Localized spoof plasmons in closed textured cavities", Appl. Phys. Lett. 104, 251601 (2014)

12. Y. J. Zhou, Q. Z. Xiao, B. J. Yang, Sci. Rep., "Spoof localized surface plasmons on ultrathin textured MIM ring resonator with enhanced resonances", 5,14819(2015)

13. B. J. Yang, Y. J. Zhou, Q.X. Xiao, "Spoof localized surface plasmons in corrugated ring structures excited by microstrip line", Opt. Express, 23, 021434 (2015)

14. Z. Gao,F. Gao, Y. Zhang and B. Zhang, "Complementary structure for designer localized surface plasmons", Appl. Phys. Lett., 107, 191103 (2015),

15. J. A. Kong, Electromagnetic Wave Theory, E M W Pub, (2000)

16. K. Chang, Microwave Ring Circuits and Antennas. New York: Wiley (1996)

17. E. A. Semouchkina, G. B. Semouchkin, M. Lanagan, and C. A. Randall, "FDTD Study of Resonance Processes in Metamaterials", IEEE Trans. Microw.Theory Tech $\mathbf{5 3}$ 4(2005)

18. S. A. Maier, Plasmonics: fundamentals and applications, Springer, (2007) 